\title{
Optimal Sensors Positioning to Detect Forest Fire Ignitions
}

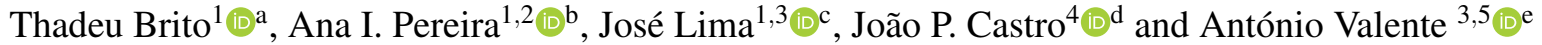 \\ ${ }^{1}$ Research Centre in Digitalization and Intelligent Robotics (CeDRI), Instituto Politécnico de Bragança, Bragança, Portugal \\ ${ }^{2}$ Algoritmi Research Centre, University of Minho, Campus de Gualtar, Braga, Portugal \\ ${ }^{3}$ INESC TEC, INESC Technology and Science, Porto, Portugal \\ ${ }^{4}$ CIMO - Centro de Investigação da Montanha, Instituto Politécnico de Bragança, Bragança, Portugal \\ ${ }^{5}$ Engineering Department, School of Sciences and Technology, UTAD, Vila Real, Portugal
}

Keywords: Wildfires, Regional Climate, Forest Fire Ignition, Ignition Detection.

\begin{abstract}
Forests have been harassed by fire in recent years. Whether by human action or for other reasons, the burned area has increased harming fauna and flora. It is fundamental to detect an ignition early in order to firefighters fight the fire minimizing the fire impacts. The proposed Forest Monitoring System aims to improve the nature monitoring and to enhance the existing surveillance systems. A set of innovative operations is proposed that will allow to identify a forest ignition and also will monitor the fauna. For that, a set of sensors are being developed and placed in the forest to transmit data and identify forest fire ignition. This paper addresses a methodology that identifies the optimal positions to place the developed sensors in order to minimize the fire hazard. Some preliminary results are shown by a stochastic algorithm that spread points to position the sensor modules in areas with a high risk of fire hazard.
\end{abstract}

\section{INTRODUCTION}

The project Forest Alert Monitoring System (SAFe) proposes to create and execute a set of innovative operations to minimize the alert time of forest fires ignitions. Consequently, these actions will contribute to the existing surveillance systems, helping the civil protection teams to decide about the development actions.

In this sense, the SAFe system proposes an intelligent procedure for monitoring situations of potential forest risk. The developed system, both hardware and software, combines distributed standalone sensor modules that will acquire and transmit several relevant data for efficient characterization of existing forest conditions. According to (Aslan et al., 2012) this information, combined with a system based on artificial intelligence, will allow the efficient and intelligent analysis of the data, promoting the creation of warnings of dangerous situations by warning several surveillance agents (for example, firefighters, civil

\footnotetext{
a (iD) https://orcid.org/0000-0002-5962-0517

b (iD) https://orcid.org/0000-0003-3803-2043

c (iD https://orcid.org/0000-0001-7902-1207

d(D) https://orcid.org/0000-0003-0647-8892

e (iD https://orcid.org/0000-0002-5798-1298
}

protection or town hall). Additionally, all this information and analysis will be made available through a web platform based on a graphical interface.

This proposal arises from the diagnosis of the unreliability of observation towers managed by human operators, which led to the testing of several technologies to improve detection capability, such as the use of fixed or mobile surveillance cameras in the visible and infrared band (Alkhatib, 2014). The accuracy of these systems is affected by terrain, time of day and weather conditions such as cloudiness, light reflection and smoke from industrial or social activities.

This work presents a methodology to identify the ideal positions to place the developed sensor modules in order to minimize the fire hazard. The data to evaluate the fire risk was obtained from national environmental agencies. Based on these data it was proposed a method to identify possible points in the Study Area with a high risk of the forest fire.

This paper is organized as follows. After an introduction in Section 1, the system architecture is presented in Section 2. Section 3 shows the selected area to address the study and Section 4 describes the sensor modules that will be spread in the forest to collect the data. Section 5 presents the LoRaWAN architecture while the preliminary results are demonstrated in 
Section 6. The conclusions and some future work are presented in Section 7.

\section{SYSTEM ARCHITECTURE}

Many applications and tools need to be combined to develop the forest monitoring system as well as the entire process involved (Singh and Sharma, 2017). Developing a strategy for joining all tools and applications is also of high importance. As a result, Figure 1 illustrates in a simplified scheme the main components of the entire SAFe project system. The following topics list the points indicated in Figure 1:

- (1) Study Area: Region to be determined for the implementation of sensor modules.

- (2) Sensor Modules: the set of sensors that make the acquisition of forest data.

- (3) LoRaWAN Gateway: Device that receives data from each sensor module scattered within the study area.

- (4) Internet: Internet communication that will facilitate real-time viewing.

- (5) LoRa Communication: LoRa-based radio frequency communication to European Union standards (Zhang et al., 2009).

- (6) Server: Component that will store all collected data, where the artificial intelligence system will also be developed to correlate data from sensor modules with external data (local scale real-time fire hazard indexes, weather data, availability fuel content and moisture content of the vegetation).

- (7) Control Center: Alerts for hazardous situations or forest fire ignitions, alert notifications are tailored to each surveillance agent in the region.

- (8) External Data: Provided by national environmental forecasting, risk and disaster response agencies, as well as local entities.

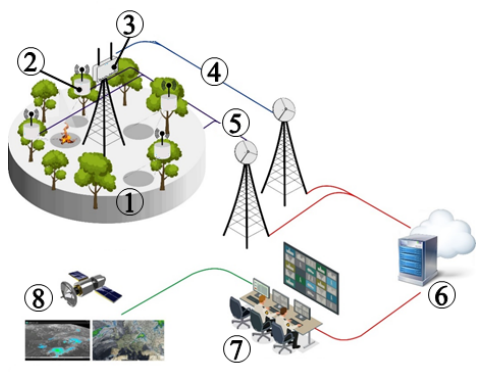

Figure 1: Illustration of the system architecture.
Among all the tools and applications developed by the SAFe project, this work will focus on the Study Area. This is outlined in Section 3.

\section{STUDY AREA}

The application of sensor modules for forest data acquisition will be implemented in the Bragança region, in the Serra da Nogueira area, as shown in Figure 2. Due to the characteristics of this forest, spreading the sensor modules across the region would be chaotic and hard to understand the data (Lloret et al., 2009). Therefore, it is necessary to develop a strategy to place the sensor modules. The chosen points must consider the data provided by the national environmental agencies.

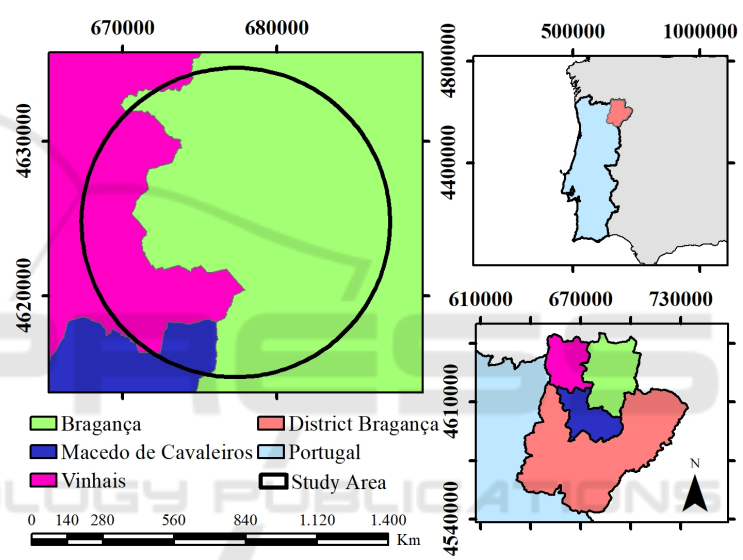

Figure 2: Geographic localization of Serra da Nogueira. Data obtained by (Copernicus, 2019).

Some factors are deterministic for the choice of these points, such as soil occupation, history and estimation of areas at flame hazard, areas that have been burned over the years, terrain elevation and forest density, among others. For the analysis of these factors, QGIS software (QGIS, 2019) is used as a visualization base and geographic data are provided by Copernicus (Copernicus, 2019). The coordinate system has the ETRS89/PT-TM06 (EPSG:3763) UTM Zone $29 \mathrm{~N}$ standard with Mercator Transverse Universal projection, the unit of measurement used is in meters. Thus, it is possible to develop a methodology that respects the characteristics of the Study Area according to the range of sensor modules, which is described in the following section. 


\section{SENSOR MODULES}

There are multitude of flame sensors on the market, the type of products found can be based on image analysis (cameras), gas detection (smoke detector), heat change (thermal cameras), radiation wave spectrum among the others. However, some methods have disadvantages during their application. For example, image analysis to detect fire ignitions may contain human failures (Chen et al., 2004), smoke sensors have high energy consumption (Baranov et al., 2015), and thermal cameras have a high installation cost (Katayama et al., 2009). Because of this, the development of sensor modules is based on low-cost sensors through radiation detection.

Figure 3 shows the prototype design of a sensor module. Figure 3 a describes the sensor module box whereas Figure $3 b$ describes the components. The central element is the Arduino Uno (1) which through LoRa communication (2) sends data from five infrared radiation sensors (3), temperature and humidity sensors (4), UV index (5), and soil moisture (6). The box is $100 \mathrm{~mm}$ long, $70 \mathrm{~mm}$ wide and $72 \mathrm{~mm}$ high. It is made in a $3 \mathrm{D}$ printer, so that printing did not waste filaments with supports. It is also water-resistant.
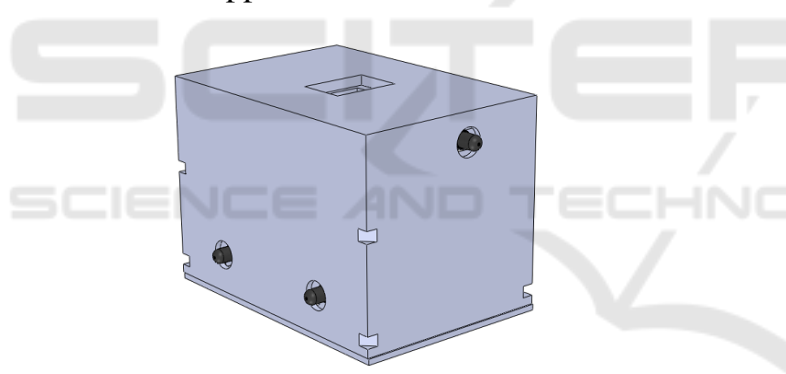

(a) Sensor module simulation

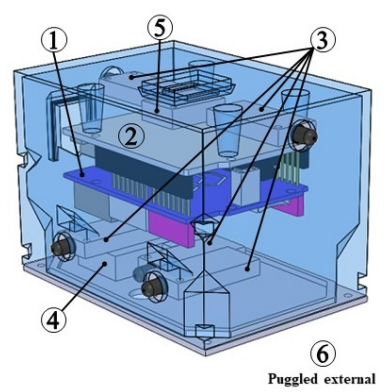

(b) Sensor module description

Figure 3: Prototype design of a sensor module.

All sensors described are used in a standard manner, i.e. without any change in the method suggested by the manufacturers. Therefore, the box can be produced and assembled for the first analysis. The final sensor module box is presented in Figure 4.

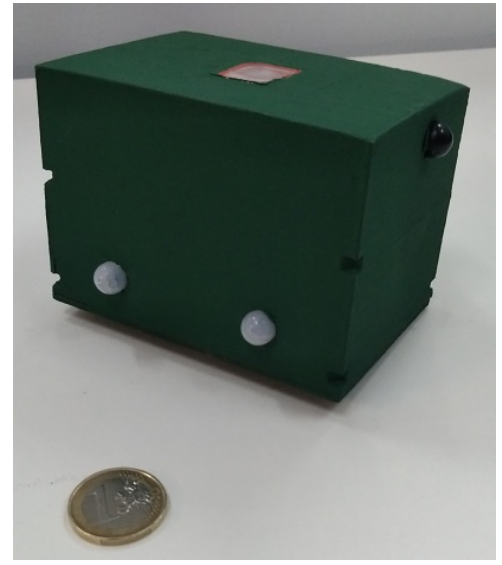

Figure 4: Real sensor module.

\section{COMMUNICATION SYSTEM}

LoRa (Long Range) is the adopted protocol to establish the connection between sensors and the central servers. More details of the communication system can be found at (Adorno et al., 2019). LoRa is a wide-area network technology that has been driven by the IoT (Internet of Things) and thus is low-power consumption devices (it enables long-range transmissions of about $10 \mathrm{~km}$ in rural areas). It is uses spread spectrum modulation techniques derived from chirp spread spectrum on license-free sub-gigahertz radio frequency bands. The developed wireless sensors network uses the $868 \mathrm{MHz}$, one of the standard frequencies for LoRa in Europe. On the upper network layer of LoRa, LoRaWAN is one of several protocols that were developed to access cloud-based services. It manages the communication between LoRaWAN gateways and end-node devices. In fact, the Industry 4.0 boosted the IoT and IIoT (Industrial Internet of Things) that makes use of LoRaWAN in several areas, such as Smart Cities, agriculture and logistics among the others.

Figure 5 shows the architecture of the LoRaWAN. The nodes, through LoRa, connect to the gateways that send the acquired data to the application server where the processing will be done. At the proposed system, the ignition detection should be done.

\section{PRELIMINARY RESULTS}

Before placing the detection prototype in the forest, some tests are required to perform in a controlled environment. Lab testing is important to ensure that the prototype is able to acquire data and send it remotely. 


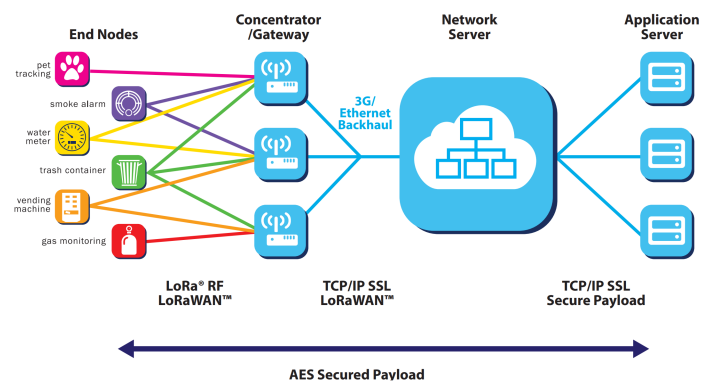

Figure 5: LoraWAN architecture. From (LoRa Alliance Technical Marketing Workgroup, 2019).

This avoids possible communication failures as well as some false alarms. In parallel, after some short analysis made from the data obtained by (Copernicus, 2019), it was possible to establish some parameters for choosing the locations that will receive the sensor modules. The following subsections describe the results obtained regarding lab testing and geographic information.

\subsection{The SAFe Box}

After all the design, 3D printing and prototype assembly, the first evaluation is to determine if the sensors can function without interruptions. Then, the prototype was turned on for 30 days collecting data within the laboratory with a 2-second collection interval. All data were recorded without interruption, ie all sensors were able to collect data at any time of the day and without large peak oscillations. Due to a large amount of data, Figure 6 shows only data collected in a single day.

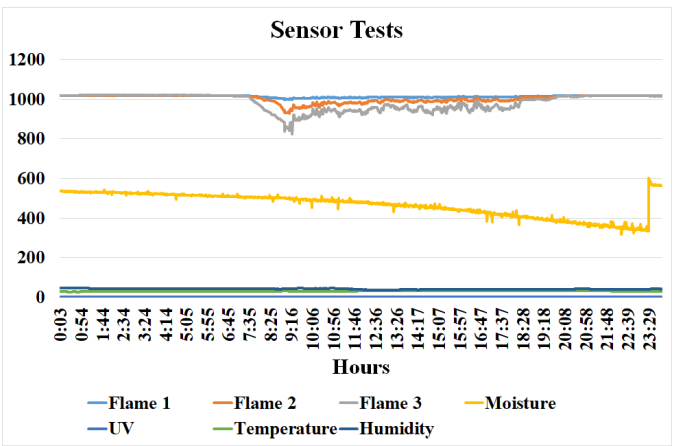

Figure 6: Chart from the collected data in one day. Values are dimensionless since the output of sensors are presented after a 10 bit Analog to digital conversion.

In the data acquisition chart presented in Figure 6, it is possible to establish the minimums and maximums values of the flame sensors, where values have ranged from 0 to 1023 (values from Arduino UNO analog input). When the sensor is close to the flame, the values tend to be close to 0 . On the other hand, when the sensor moves away from the flame, the values tend to approach 1023 . The sensitivity of the sensor varies with distance, intensity and flame volume. By simulating the forest fire ignition with a candle, it is possible to detect the presence of fire at about 5 $\mathrm{m}$. The same process occurs for the soil moisture sensors, that is, when the soil is wet the acquired data are close to 0 and when the soil is dry the values are close to 1023 . The UV index sensor provided low values due to the lack of light exposure once the tests were performed indoors.

With the first analysis, it was observed that the sensors did not suffer interruptions in data collection, so the use of LoRa communication becomes viable for the SAFe project approach. Then, the second analysis evaluates prototype fixation in real situations, that is, being fixed to a tree trunk in the Study Area region. Figure 7 shows the mounting and placement of sensors on the stem of the tree. Note that the orientation of the sensors is free, that means there are no barriers to the capture of infrared spectra. Another factor is wireless, all data collection was done with LoRa communication.

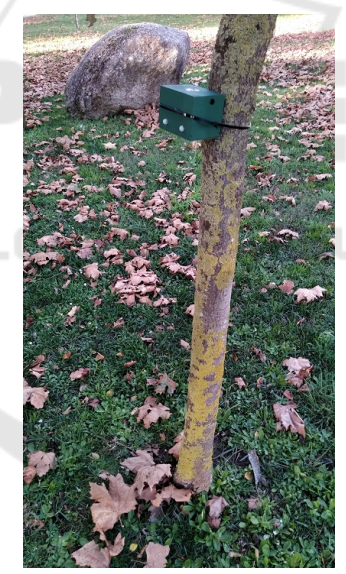

(a) Prototype fixation.

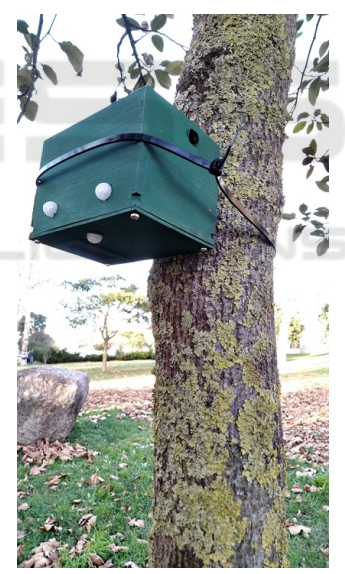

(b) Placement of sensors.
Figure 7: SAFe box acquiring data from Serra da Nogueira forest.

As mentioned earlier, installing modules on all tree trunks in a forest can become a daunting and costly task. Finding the ignition point of fire can dramatically slow down the work of this task. Therefore, the following subsection 6.2 demonstrates the strategy for finding the highest fire risk points.

\subsection{Fragments of Study Case}

The total coverage area of the monitoring system is expected to be a radial $10 \mathrm{~km}$, so installing the mod- 
ules with a distance of $5 \mathrm{~m}$ between them along this circular $10 \mathrm{~km}$ area will be chaotic and laborious. To solve the problem of territorial extension of $10 \mathrm{~km}$ not becoming chaotic for data collection modules and communication, it was determined that a possible approach is to separate the whole terrain into small fragments. These fragments will be divided into a maximum of $1 \mathrm{~km}$ of diameter. In this way, it is possible to understand the critical points of each fragment, to position the sensors according to local resources and to prepare the communication infrastructure.

Before considering the total fragmentation to 10 $\mathrm{km}$, it is necessary to study the region according to some parameters that contribute to the likelihood of forest fires and then to define the best central point to start the study. This central point should contain an intersection area between the parameters that contribute to the likelihood of forest ignition. The type of soil occupation contributes to the incidence of wildfire (Verde, 2010), as shown in Figure 8 (Bragança region has a mixed occupation).
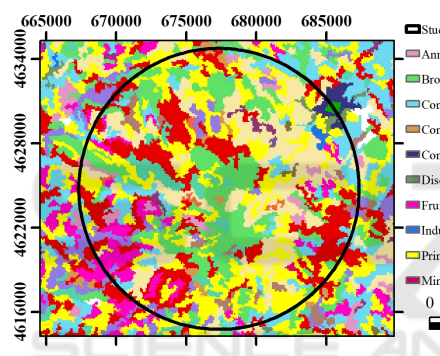

Figure 8: Different type of soil occupation activities. Data obtained by (Copernicus, 2019).

Particularly, it is important to analyse the data of transitional woodland-shrub, non irrigated arable land, broad-leaved forest, fire hazard, the history of the burned area over the last years, the soil relief and tree density among others. With the analysis made from Figure 9, it is possible to notice this relationship between the type of land occupation with the fire hazard estimate and the burned areas over the last years (comparing Figure 9a with Figure 9b).

Since the relief interferes with soil moisture, the vegetation is drier in higher altitudes and consequently prone to ignition of flames. Figure 10a and Figure $10 \mathrm{~b}$ demonstrate the occurrence of a high forest density in the high altitude regions. Therefore, the center point should also contain a high altitude relative to the other points within $10 \mathrm{~km}$ along with a high forest density.

By comparing the data displayed in QGIS, shown in Figures 9 and 10, it is possible to determine some points that comply with the parameters that increase the likelihood of forest fire. The central region was

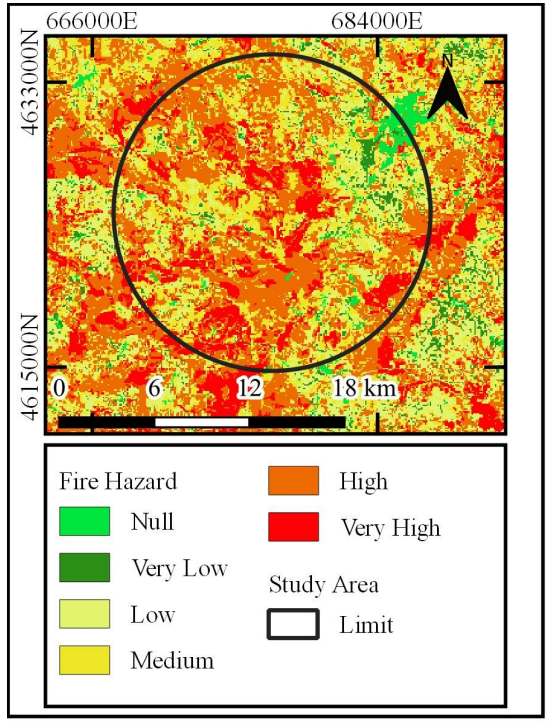

(a) Fire Hazard. Data obtained by (ICNF, 2019).

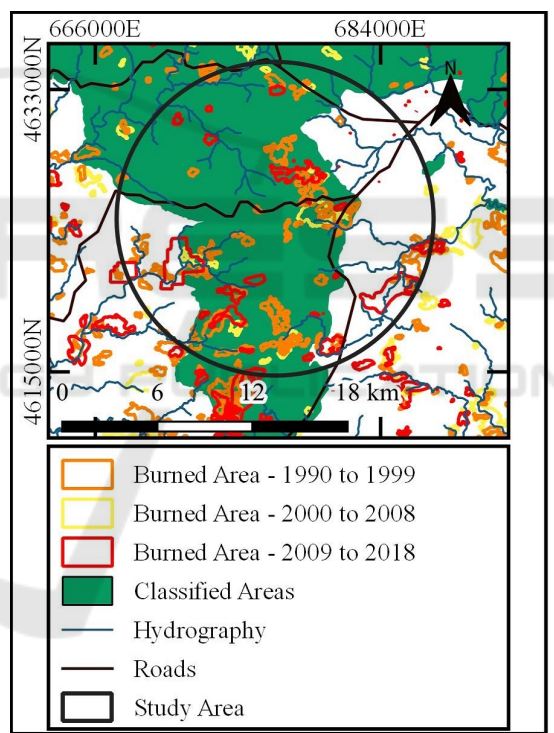

(b) Burned areas over the last years. Data obtained by (Copernicus, 2019).

Figure 9: The Study Area with the fire risk estimate data and the burned areas information. Data obtained by (Copernicus, 2019).

defined with coordinates $(105327,232506)$ and altitude near 1050 m, shown by Figure 11, in Serra da Nogueira.

From this central point as a reference, the Fire Hazard layer is activated and by selecting between the values 0 and 5 it is possible to choose only the regions of greatest interest, i.e. the regions with danger values 4 and 5. Thus, some regions are not considered for the 


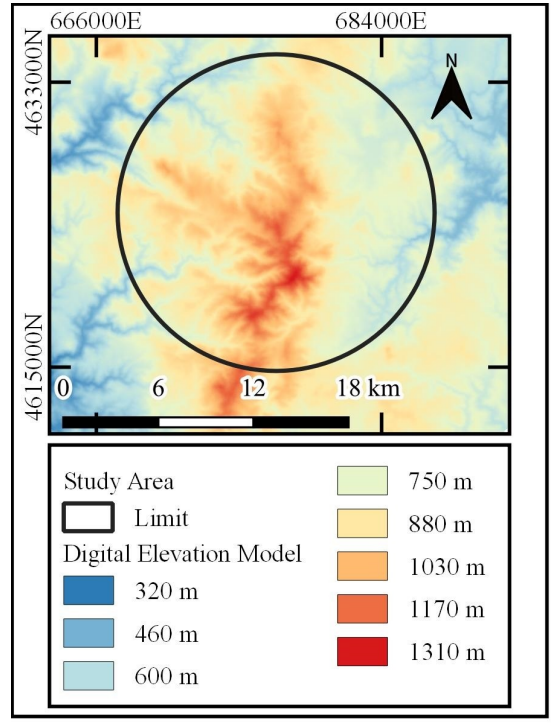

(a) Soil relief. Data obtained by (Copernicus, 2019).

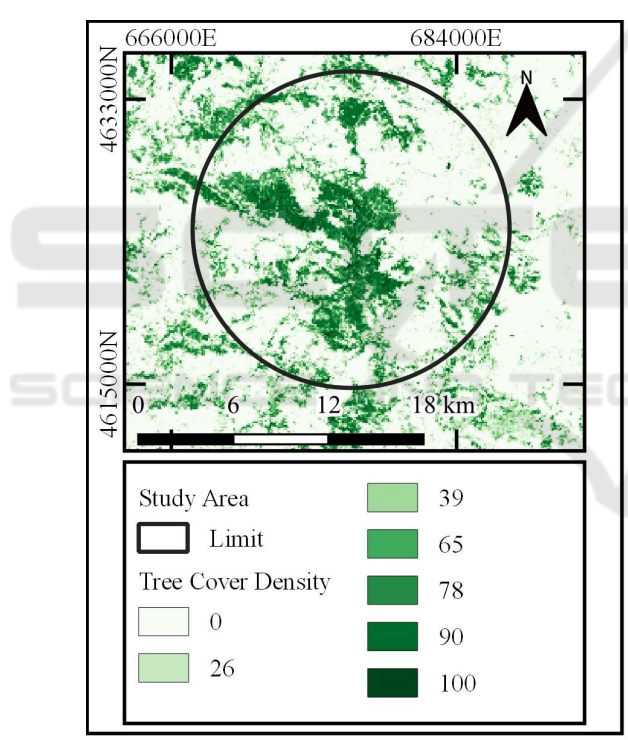

(b) Tree cover density. Data obtained by (Copernicus, 2019).

Figure 10: The Study Area Figure presents the soil relief with forest density in the Serra da Nogueira. Data obtained by (Copernicus, 2019).

selection of place the sensor modules, which makes the process a little less chaotic. Figure 12 demonstrates the fire hazard layer at the boundaries of the $10 \mathrm{~km}$ radius from the center point.

Figure 12 addresses $1 \mathrm{~km}$ region around the defined center point, shown in Figure 13a. The presented area can be zoomed in and the developed algorithm can be applied to have as base the fire hazard

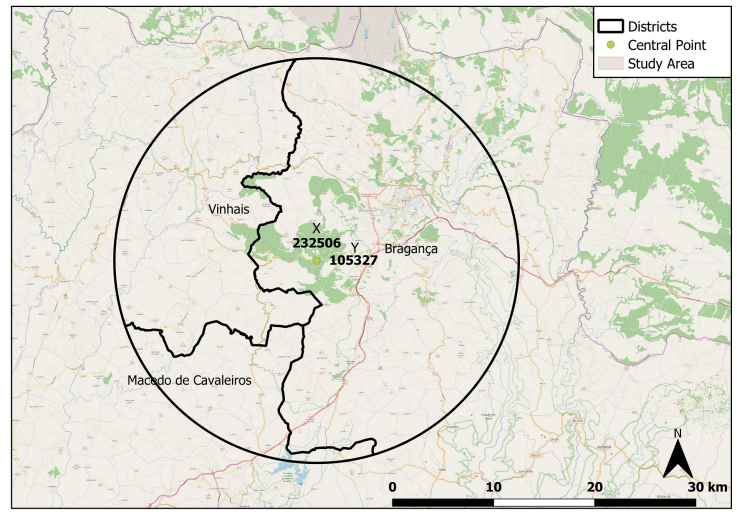

Figure 11: Central point in Bragança region.

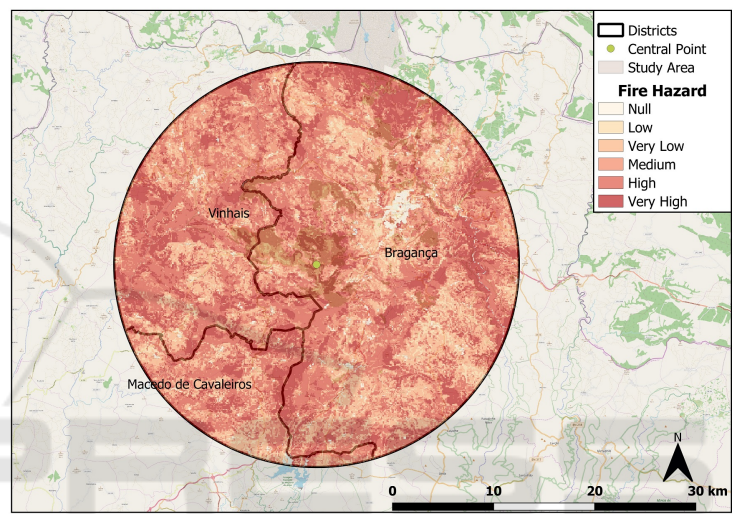

Figure 12: Fire hazard layer along the Study Area. Data obtained by (ICNF, 2019)

map. The data to feed the algorithm was extracted from the $1 \mathrm{~km}$ radius fire hazard layer from the center point, presented in Figure 13b. As already mentioned, values between 0 and 3 do not present great possibilities for forest ignition, so only regions with values 4 and 5 are selected (Figure 13c).

The selected regions are transformed into polygon formats since QGIS cannot access the data in Raster format. Then each region generates a set of polygons that are entered separately in the random point insertion algorithm. For the distribution of random points, it was set as 10000 maximum points for each polygon and with a distance between each point of $5 \mathrm{~m}$. The operation of random point distribution is shown in Algorithm 1.

After the algorithm determines the location of the random points, a point mask is created over the polygons that have been inserted as a fastening space. Figure 14 shows the result of this mask over the region with hazard values 4 and 5 . Note that in both Figure 14a and Figure 14b the entire fire hazard region has been completely populated, which validates the approach of the implemented algorithm. 


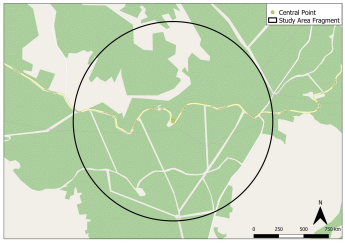

(a) Study Area Fragment. (b) Fire hazard layer along the Fragment.
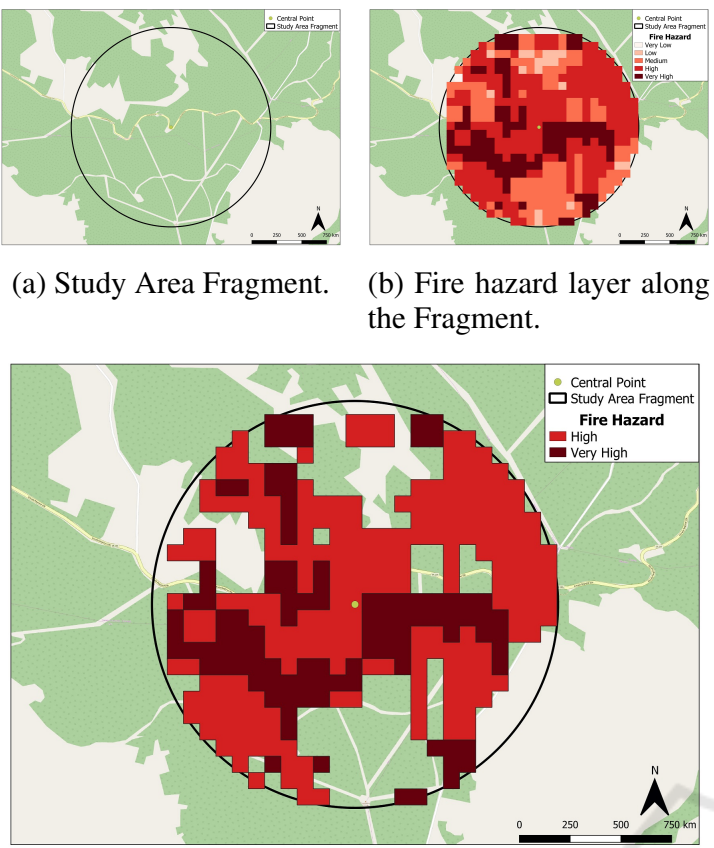

(c) Selected regions with High and Very High levels.

Figure 13: Sequence of images demonstrating the selection of regions with the highest risk of forest ignition within the Study Area Fragment. Data obtained by (ICNF, 2019).

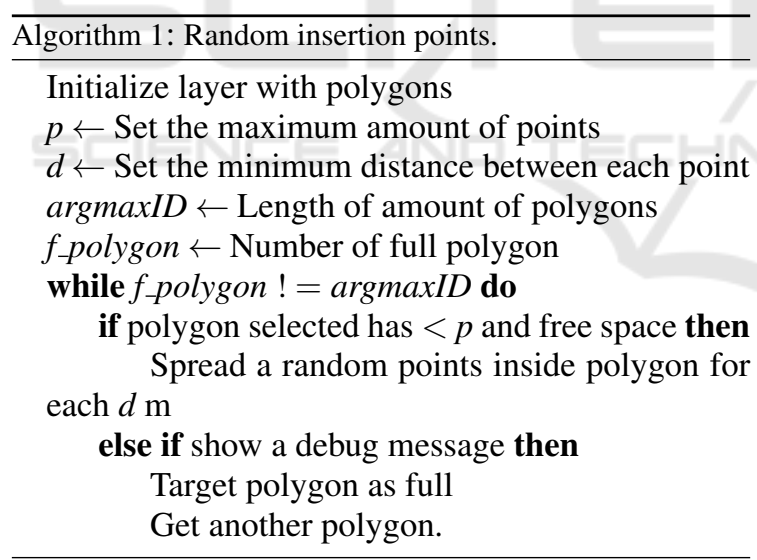

At the end of each presented Algorithm run, the amount of time is reported in log format. It is also collected from each generated mask the total random points entered. This information is shown in the Table 1.

Table 1: Results of the algorithm.

\begin{tabular}{ccc}
\hline Layer & Points & Time [s] \\
\hline Region with fire hazard values 4 & 24601 & 460.30 \\
Region with fire hazard values 5 & 18482 & 464.16 \\
\hline
\end{tabular}

By zooming the region a bit more, it can be found a better distribution of the points, as shown in the set

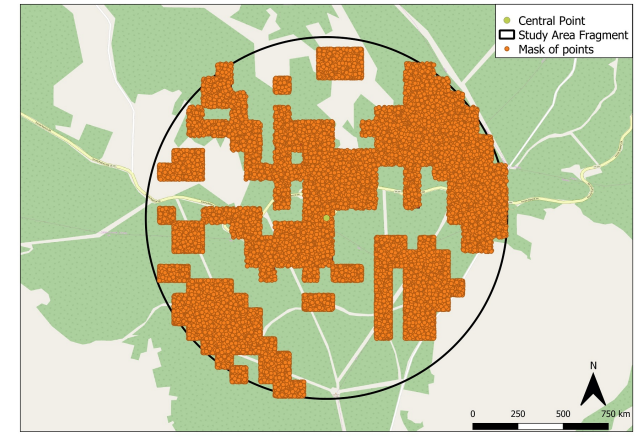

(a) Points generated for regions with fire hazard values 4 .

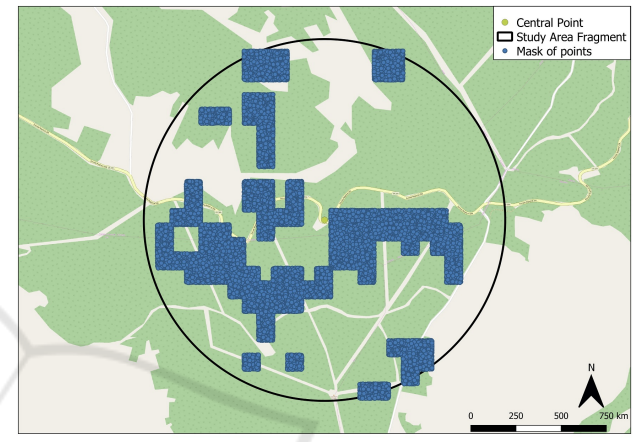

(b) Points generated for regions with fire hazard values 5 .

Figure 14: Mask of points generated by the Algorithm 1 according to each region inserted as a fixation space.

of images of Figure 15. When using the QGIS measurement tool, it is noted that the points are $5 \mathrm{~m}$ away to each other and are also limited by the chosen regions. These identified points will be the candidates to place a sensor module to acquire the data.

\section{CONCLUSIONS AND FUTURE WORK}

Monitoring forests can warn of possible fire ignition that early detected help combat teams to minimize fire impacts. The presented Forest Monitoring System aimed to contribute to support the actual surveillance systems by implementing a set of innovative operations that allow to identify a forest ignition based on a set of sensors positioned in the forest. This work presented a methodology to propose a set of locations to install the sensor boxes. The results are promising since the data gathered from the installed sensors boxes prototypes are operational. Moreover, a first version of the prototype sensor box was developed, tested and validated in laboratory and real scenarios 


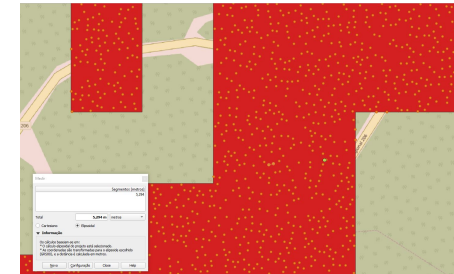

(a) Zoomed view of the region with fire hazard 4 values over the generated dot mask.

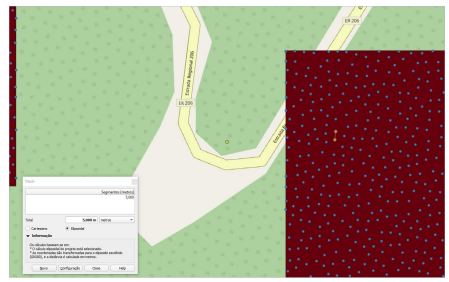

(b) Zoomed view of the region with fire hazard 5 values over the generated dot mask.

Figure 15: Magnified view of each chosen region with distance measurements between each point made via QGIS.

transmitting the acquired data to the central processing. As future work, the forest data will be collected and analysed with artificial intelligent algorithm in order to identify data patterns and alerts to the control servers, triggering in the case of an ignition detection.

\section{ACKNOWLEDGEMENTS}

This work has been supported by Fundação La Caixa and FCT — Fundação para a Ciência e Tecnologia within the Project Scope: UIDB/5757/2020.

\section{REFERENCES}

Adorno, D., Soares, S., Lima, J., and Valente, A. (2019). Evaluation of lp-wan technologies for fire forest detection systems. In ALLSENSORS 2019, The Fourth International Conference on Advances in Sensors, Actuators, Metering and Sensing, pages 49-53. IARIA Conference.

Alkhatib, A. A. (2014). A review on forest fire detection techniques. International Journal of Distributed Sensor Networks, 10(3):597368.

Aslan, Y. E., Korpeoglu, I., and Ulusoy, Ö. (2012). A framework for use of wireless sensor networks in forest fire detection and monitoring. Computers, Environment and Urban Systems, 36(6):614-625.

Baranov, A., Spirjakin, D., Akbari, S., and Somov, A. (2015). Optimization of power consumption for gas sensor nodes: A survey. Sensors and Actuators A: Physical, 233:279-289.

Chen, T.-H., Wu, P.-H., and Chiou, Y.-C. (2004). An early fire-detection method based on image processing. In 2004 International Conference on Image Processing, 2004. ICIP'04., volume 3, pages 1707-1710. IEEE.

Copernicus (2019). European union's earth observation programme. https://www.copernicus.eu. Accessed November, 2019.

ICNF (2019). Instituto de conservação da natureza e das florestas. https://www.icnf.pt. Accessed November, 2019.

Katayama, H., Naitoh, M., Suganuma, M., Harada, M., Okamura, Y., Tange, Y., and Nakau, K. (2009). Development of the compact infrared camera (circ) for wildfire detection. In Remote Sensing System Engineering II, volume 7458, page 745806. International Society for Optics and Photonics.

Lloret, J., Garcia, M., Bri, D., and Sendra, S. (2009). A wireless sensor network deployment for rural and forest fire detection and verification. sensors, 9(11):8722-8747.

LoRa Alliance Technical Marketing Workgroup, L. (2019). technical overview of lora and lorawan. help@ lora-alliance.org. November, 2019.

QGIS (2019). A free and open source geographic information system. https://qgis.org. Accessed November, 2019.

Singh, P. K. and Sharma, A. (2017). An insight to forest fire detection techniques using wireless sensor networks. In 2017 4th International Conference on Signal Processing, Computing and Control (ISPCC), pages 647653. IEEE.

Verde, J. C. (2010). Avaliação da perigosidade de incêndio florestal. $\mathrm{PhD}$ thesis, University of Lisbon.

Zhang, J., Li, W., Yin, Z., Liu, S., and Guo, X. (2009). Forest fire detection system based on wireless sensor network. In 2009 4th IEEE conference on industrial electronics and applications, pages 520-523. IEEE. 